\title{
Effectiveness of introducing a 20-gauge core biopsy needle with a core trap in EUS-FNA/B for diagnosing pancreatic cancer
}

\author{
Shunsuke Watanabe ${ }^{1}$, Jun Miyoshi ${ }^{1 *}$, Masao Toki ${ }^{1}, K^{\prime}$ omei Kambayashi ${ }^{1}$, Shuichi Kitada ${ }^{1}$, Takeshi Nosaka , \\ Tomoyuki Goto ${ }^{1}$, Hirotaka Ota ${ }^{1}$, Kazushige Ochiai ${ }^{1}$, Koichi Gondo ${ }^{1}$, Nobuhito Ikeuchi ${ }^{2}$, Shujiro Tsuji ${ }^{2}$, \\ Kenji Nakamura ${ }^{1,3}$, Junji Shibahara $^{4}$ and Tadakazu Hisamatsu ${ }^{1 *}$ (i)
}

\begin{abstract}
Background: Endoscopic ultrasound-guided fine-needle aspiration/biopsy (EUS-FNA/B) is a standard method for pathological diagnosis of pancreatic solid lesions. The EchoTip ProCore 20G ${ }^{\circledR}$ (PC20), a 20-gauge biopsy needle with a forward-bevel core trap, has been available in Japan since 2015.

Methods: We compared the efficacy of the PC20 with that of the EchoTip ProCore 22G ${ }^{\circledR}$ (PC22) and Acquire 22G ${ }^{\circledR}$ (AC22) in EUS-FNA/B for diagnosing pancreatic cancer. This retrospective study included 191 patients with pancreatic cancer who underwent EUS-FNA/B using the PC20, PC22, or AC22 at our facility from April 2013 to October 2019. We investigated the patients' clinical characteristics and the diagnostic accuracy and safety of each needle.

Results: A sufficient stroke length of puncture was secured in all patients. The maximum length under EUS was shorter with the AC22 $(22.1 \pm 2.2 \mathrm{~mm})$ than PC20 $(30.6 \pm 0.7 \mathrm{~mm}, p<0.01)$ and $\mathrm{PC} 22(30.3 \pm 0.8 \mathrm{~mm}, p<0.01)$. The histological accuracy was $96.4 \%$ with the PC20 but only $58.8 \%$ with the PC22 (adjusted $p$ ( $p$-adj) $<0.0001$ ) and $75.0 \%$ with the AC22 ( $p$-adj=0.06). The diagnostic accuracy of the combination of histology and cytology was $96.4 \%$ with the PC20, while it was $72.1 \%$ with the PC22 ( $p$-adj $<0.0001)$ and $91.7 \%$ with the AC22 ( $p$-adj $>0.99)$. One patient $(0.9 \%)$ in the PC20 group developed mild pancreatitis, but no adverse events occurred with the other needles.
\end{abstract}

Conclusions: The PC20 showed better diagnostic capability than the PC22. The diagnostic efficacy was similar between the PC20 and AC22. The high histological accuracy of the PC20 could be advantageous for lesions in which histological assessment is critical.

Keywords: Pancreatic cancer, Endoscopic ultrasound-guided fine needle aspiration/biopsy, ProCore 20-gauge needle, Diagnostic accuracy

\section{Background}

Pancreatic cancer is a highly aggressive tumor with a poor prognosis [1-5]. The age-standardized incidence rate of pancreatic cancer increased by $1.03 \%$ per year

\footnotetext{
*Correspondence: jmiyoshi@ks.kyorin-u.ac.jp; thisamatsu@ks.kyorin-u.ac.jp 1 Department of Gastroenterology and Hepatology, Kyorin University School of Medicine, 6-20-2 Shinkawa, Mitaka-shi, Tokyo 181-8611, Japan Full list of author information is available at the end of the article
}

from 1973 to 2014 [6]. Changes in environmental and behavioral factors rather than genetic factors are thought to be related to the worldwide increase in pancreatic cancer $[7,8]$. Differences in lifestyle (e.g., high-calorie diet and high body mass index), exposure to environmental risk factors (e.g., smoking), and accessibility to medical services including more efficient diagnostic tools might be associated with the variability in the mortality of pancreatic cancer among countries $[7,9,10]$. 
Pancreatic cancer is typically found as a pancreatic solid lesion. Pancreatic solid lesions include pancreatic malignant lesions such as pancreatic cancer, pancreatic neuroendocrine tumors, and metastatic pancreatic cancer as well as benign lesions such as autoimmune pancreatitis and mass-forming pancreatitis. Therefore, prompt and accurate diagnosis of pancreatic cancer is critical to determine the most appropriate therapeutic strategies. For this purpose, pathological diagnosis with minimal invasion is crucial. Endoscopic ultrasound-guided fineneedle aspiration/biopsy (EUS-FNA/B) is the standard method for sampling pathological specimens from a pancreatic solid lesion. The reported diagnostic sensitivity, specificity, and accuracy are $91.7-93.4 \%, 100 \%$, and $92.3 \%$ to $94.4 \%$, respectively $[11,12]$. Various factors, including the type of puncture needle, puncture route, number of passes, tumor size, and experience of the endoscopist and cytopathologist, can impact the accuracy of diagnosis [13-15]. Several types of puncture needles with various characteristics (e.g., thickness and needle-tip shape) are commercially available [16-18]. Also, the needles are categorized into FNA needles and FNB needles and the systematic review by Li et al. [19] presented FNB are superior. The volume of a specimen obtained by core needle biopsy can depend on the thickness of the puncture needle; i.e., a needle with a larger diameter can collect a larger biopsy specimen [20]. A higher sample volume contributes to a more accurate pathological diagnosis [20]. However, in the clinical setting, a thicker needle makes the puncture procedure more difficult, particularly during transduodenal puncture in which the steep up-angle of the scope results in less flexibility and greater friction for the needle sheath. Moreover, this steep up-angle can distort the puncture needle, necessitating additional needles for a subsequent puncture. Given these facts, a needle that is flexible but can collect a sufficient volume of specimens for pathological assessment is needed for pancreatic core needle biopsy.

The EchoTip ProCore ${ }^{\circledR}$ needle (Wilson Cook Medical Inc., Winston-Salem, NC, USA) has a hollowed-out core trap on the side of the needle. This core trap system is designed to improve the acquisition of core biopsy specimens. Sterlacci et al. [21] reported that EUS tissue sampling using a 22-gauge (22G) needle with a core trap increased the accuracy of pathological diagnosis for abdominal masses compared with using a 22G aspiration needle. EchoTip ProCore ${ }^{\circledR}$ needles of various diameters are currently available. In Japan, the EchoTip ProCore $22 \mathrm{G}^{\circledR}$ (PC22) was approved for pancreatic EUS-FNA/B in March 2012 and the EchoTip ProCore 20G ${ }^{\circledR}$ needle (PC20) in December 2015. Notably, the PC20 has an anterograde, forward-bevel core trap, while the $\mathrm{PC} 22$ has a retrograde, reverse-bevel core trap. Additionally, the PC20 employs a new coil sheath resulting in greater flexibility of the needle, making procedures involving the duodenum easier. A recent study using porcine livers showed preferable tissue acquisition with the PC20 [22]. However, whether the PC20 can improve the diagnostic capability of EUS-FNA/B for pancreatic cancer in the real-world clinical setting has not been proven. In the present study, we retrospectively assessed the diagnostic efficacy and safety of the PC20 in patients with pancreatic cancer.

\section{Methods \\ Patients}

In this retrospective study, we investigated the clinical database of Kyorin University Hospital. We analyzed 191 patients who were diagnosed with pancreatic cancer and underwent their first EUS-FNA/B with the PC20, PC22, or Acquire 22 $\mathrm{G}^{\circledR}$ (AC22) (Boston Scientific, Natick, MA, USA) for pancreatic solid lesions from April 2013 to October 2019. The final diagnosis of pancreatic cancer was confirmed by pathological diagnosis with EUSFNA/B, percutaneous pancreatic biopsy, open biopsy, or ascites cytology. Some patients underwent EUS-FNA/B multiple times. Our board-certified pathologists at Kyorin University Hospital assessed both cytology and histology. The pathological diagnosis of each specimen was confirmed by multiple board-certified pathologists. A histological specimen from each pass was assessed. The diagnosis of pancreatic cancer was made when adenocarcinoma was histologically diagnosed in a specimen. In cytologic assessment, a specimen with class IV and V cells was diagnosed as pancreatic cancer. For specimens that could not be definitively diagnosed by these procedures, the diagnosis of pancreatic cancer was made based on the patient's clinical course with scheduled imaging tests during the 12-month period following EUS-FNA/B.

\section{Ethics statement}

The present study was approved by the Institutional Review Board of Kyorin University School of Medicine (IRB No. 1099) on 27 March 2018. This study was performed in accordance with the guidelines of the Declaration of Helsinki. Informed consent was obtained using an opt-out method.

\section{Procedure of EUS-FNA/B}

All patients underwent conscious sedation by administration of midazolam (Maruishi Pharmaceutical, Osaka, Japan) and pentazocine (Maruishi Pharmaceutical). The EUS-FNA/B procedures were performed using a linear array echoendoscope (GF-UCT260 ${ }^{\circledR}$; Olympus Medical Systems, Tokyo, Japan or EG-580UT ${ }^{\circledR}$; Fujifilm Medical, Tokyo, Japan) connected to a processor featuring a 
color Doppler function (EU-ME1, $2^{\circledR}$; Olympus Medical Systems or SU-1 ${ }^{\circledR}$; Fujifilm Medical, respectively). EUSFNA/B was performed by physicians with $>5$ years of experience in EUS or by fellows in our department under the supervision of these experienced physicians. The target lesion for FNA/B was visually confirmed with EUS, and color Doppler was used to evaluate the vascularity along the puncture route. The stroke length is defined as the length of a tumor measured by EUS at the puncture line. The pancreatic solid tumor was punctured through the transgastric or transduodenal route with the PC20, $\mathrm{PC} 22$, or AC22. After the needle reached the target, the stylet was removed and suction was applied using a 10 -ml syringe. For every puncture, 20 to-and-fro movements were employed. After removing the needle, the stylet was inserted and a sample was taken and placed on a glass slide. The filamentous sample was immersed in a $10 \%$ formalin solution for histological diagnosis, and the remaining specimen on the glass slide was smeared for cytological diagnosis. Following each pass, the endoscopists assessed the specimens by macroscopic on-site evaluation (MOSE). If a large amount of bleeding occurred at the first pass, a second pass was performed by changing the suction method to the slow-pull method. If the specimens were macroscopically insufficient, more passes were performed (maximum of six passes).

\section{MOSE}

The result of MOSE was classified into three categories. In a filamentous sample of $\geq 5 \mathrm{~mm}$, white tissue was defined as an adequate specimen and a reddish sample containing a large amount of blood as a clotted specimen. A sample of $<5 \mathrm{~mm}$ was defined as a fragment specimen.

\section{Accuracy of diagnosis of pancreatic cancer}

Patients who were diagnosed with pancreatic cancer by the first EUS-FNA/B were defined as those who had obtained an "accurate diagnosis" by EUS-FNA/B. The accuracy of EUS-FNA/B was calculated as the proportion of patients who obtained an accurate diagnosis by EUSFNA/B among all patients with pancreatic cancer.

\section{Statistical analysis}

The chi-squared test and Fisher's exact test were performed to compare patient backgrounds (sex, use of antiplatelet/anticoagulant agents, comorbid diabetes mellitus, smoking, gastric surgical history, location of pancreatic tumor, and puncture line), the accuracy of the pancreatic cancer diagnosis by EUS-FNA/B, the incidence of needle distortion, and the incidence of adverse events among the different core biopsy needles. For multiple comparisons of diagnostic accuracy, Bonferroni correction was employed to calculate the adjusted $p$ value ( $p$-adj). The Kruskal-Wallis test and Dunn's test were performed to compare the patients' age, maximum diameter of the tumor measured on contrast-enhanced computed tomography images, maximum stroke length for puncture measured on EUS images, puncture stroke length, and number of passes between needles. Statistical significance was defined as $p<0.05$. GraphPad Prism version 8.1.2 (GraphPad Software, San Diego, CA, USA) was used for the statistical analyses.

\section{Results \\ Patients' backgrounds}

We analyzed 191 patients who underwent their first EUSFNA/B with the PC20, PC22, or AC22 from April 2013 to October 2019 at Kyorin University Hospital and were eventually diagnosed with pancreatic cancer. In Japan, the PC20, PC22, and AC22 were approved for EUS-FNA/B for pancreatic solid lesions by the Ministry of Health, Labour and Welfare in December 2015, March 2012, and October 2016, respectively. Among the 191 patients, the first EUS-FNA/B was performed in 53 patients from April 2013 to November 2015, in 13 patients from December 2015 to September 2016, and in 125 patients from October 2016 to October 2019. The PC20, PC22, and AC22 were used for 111,68 , and 12 patients, respectively. The patients' characteristics are shown in Table 1. There was no significant difference in sex, age, tumor location, or puncture route among the needles. However, the maximum diameter of the tumor measured on contrastenhanced computed tomography images was significantly different among the needles (PC20, 37.9 $\pm 1.2 \mathrm{~mm}$; PC22, 32.9 $\pm 1.2 \mathrm{~mm}$; AC22, $22.7 \pm 2.6 \mathrm{~mm} ; p<0.0001)$. Although a sufficient stroke length of puncture was secured in all patients, the maximum stroke length for puncture measured on EUS images was shorter with the AC22 $(22.1 \pm 2.2 \mathrm{~mm})(p<0.01$ and $p<0.01$ compared with $\mathrm{PC} 20$ and $\mathrm{PC} 22$, respectively), while there was no significant difference between the PC20 and PC22 $(30.6 \pm 0.7$ and $30.3 \pm 0.8 \mathrm{~mm}$, respectively; $p>0.99)$. The number of passes was significantly higher with the PC22 $(4.1 \pm 0.7)$ than PC20 $(3.7 \pm 0.9, p<0.01)$ and AC22 $(3.5 \pm 0.6, p<0.05)$.

\section{Diagnostic accuracy of EUS-FNA/B needles for pancreatic cancer}

The diagnostic accuracy for pancreatic cancer with the PC20 was $96.4 \%$ (107/111), 81.1\% (90/111), and 96.4\% $(107 / 111)$ by histology, cytology, and the combination of histology and cytology, respectively (Table 2). The accuracy with the PC22 was 58.8\% (40/68), 63.2\% $(43 / 68)$, and $72.1 \%(49 / 68)$ and that with the AC22 was $75.0 \%(9 / 12), 83.3 \%(10 / 12)$, and $91.7 \%(11 / 12)$, respectively (Table 2 ). The diagnostic accuracy with the PC20 
Table 1 Patients characteristics

\begin{tabular}{|c|c|c|c|c|}
\hline & PC20 & $\mathrm{PC} 22$ & $\mathrm{AC} 22$ & $p$ value \\
\hline Number of patients & 111 & 68 & 12 & \\
\hline Age (years) (median, range) & $70(40-87)$ & $71(47-85)$ & $73(52-80)$ & $0.69^{\S}$ \\
\hline Sex & & & & $0.35^{\#}$ \\
\hline Male & $59(53.1 \%)$ & $37(54.4 \%)$ & $9(75 \%)$ & \\
\hline Female & $52(46.9 \%)$ & $31(45.6 \%)$ & $3(25 \%)$ & \\
\hline Anti-platelet/anti-coagulant agents & $12(10.8 \%)$ & $11(16.2 \%)$ & $3(25 \%)$ & $0.29^{\#}$ \\
\hline Diabetes Mellitus & $32(28.8 \%)$ & $24(21.6 \%)$ & $2(16.7 \%)$ & $0.37^{\#}$ \\
\hline Smoking & $16(14.4 \%)$ & $8(11.8 \%)$ & $3(25 \%)$ & $0.47^{\#}$ \\
\hline Post-operative stomach & $1(0.9 \%)$ & $1(1.47 \%)$ & $0(0 \%)$ & $0.87^{\#}$ \\
\hline Location of the pancreatic tumor & & & & $0.88^{\#}$ \\
\hline Head & $33(29.7 \%)$ & $19(28.0 \%)$ & $5(41.7 \%)$ & \\
\hline Body & $56(50.5 \%)$ & $37(54.4 \%)$ & $5(41.7 \%)$ & \\
\hline Tail & 22 (19.8\%) & $12(17.6 \%)$ & $2(16.7 \%)$ & \\
\hline Puncture line & & & & $0.71^{\#}$ \\
\hline Trans gastric & $85(76.6 \%)$ & $50(73.5 \%)$ & $8(66.7 \%)$ & \\
\hline Trans duodenal & $26(23.4 \%)$ & $18(26.5 \%)$ & $4(33.3 \%)$ & \\
\hline Maximum diameter of tumor (mm) (mean \pm SEM) & $37.9 \pm 1.2^{*, \$ \$ \$ \$}$ & $32.9 \pm 1.2^{\prime}$ & $22.7 \pm 2.6$ & $<0.0001^{\S}$ \\
\hline Maximum stroke length of puncture $(\mathrm{mm})($ mean $\pm \mathrm{SEM})$ & $30.6 \pm 0.7^{\$ \$}$ & $30.3 \pm 0.8^{\mathbb{1 9}}$ & $22.1 \pm 2.2$ & $<0.01^{\S}$ \\
\hline Number of passes & $3.7 \pm 0.9^{* *}$ & $4.1 \pm 0.7^{\mathbb{1}}$ & $3.5 \pm 0.6$ & $<0.01^{\S}$ \\
\hline
\end{tabular}

PC20 EchoTip ProCore 20G, PC22 EchoTip ProCore 22G, AC22 Acquire 22G

\# Chi-squared test among PC20, PC22, and AC22

$\S$ Kruskal-Wallis test among PC20, PC22, and AC22

* $p<0.05$ and ** $p<0.01$, Dunn's test; PC20 versus PC22

\$\$ $p<0.01$ and ${ }^{\$ \$ \$} p<0.0001$, Dunn's test; PC20 versus AC22

" $p<0.05$ and " $p<0.01$, Dunn's test; PC22 versus AC22

Table 2 Diagnostic accuracy for pancreatic cancer

\begin{tabular}{lllll}
\hline & PC20 & PC22 & AC22 & p value \\
\hline Histology & $96.4 \%^{++\dagger}$ & $58.8 \%$ & $75.0 \%$ & $<0.0001^{\#}$ \\
& $(107 / 111)$ & $(40 / 68)$ & $(9 / 12)$ & \\
Cytology & $81.1 \%^{\dagger}$ & $63.2 \%$ & $83.3 \%$ & $<0.05^{\#}$ \\
& $(90 / 111)$ & $(43 / 68)$ & $(10 / 12)$ & \\
Combination of histol- & $96.4 \%{ }^{++\dagger}$ & $72.1 \%$ & $91.7 \%$ & $<0.0001^{\#}$ \\
ogy and cytology & $(107 / 111)$ & $(49 / 68)$ & $(11 / 12)$ & \\
\hline
\end{tabular}

PC20 EchoTip ProCore 20G, PC22 EchoTip ProCore 22G, AC22 Acquire 22G

\# Chi-squared test among PC20, PC22, and AC22

${ }^{\dagger}$ adjusted $p<0.05$ and ${ }^{\text {t+t+ }}$ adjusted $p<0.0001$, Fisher's exact test followed by Bonferroni correction; $\mathrm{PC} 20$ versus $\mathrm{PC} 22$

by histology, cytology, and the combination of histology and cytology was significantly better than that with the PC22 ( $p$-adj $<0.0001, p$-adj $<0.05$, and $p$-adj $<0.0001$, respectively), while there was no significant difference between the $\mathrm{PC} 20$ and $\mathrm{AC} 22$ ( $p$-adj $=0.06, p$-adj $>0.99$, and $p$-adj $>0.99$, respectively). The addition of cytological assessment to histological assessment improved the diagnostic accuracy with the PC22 and AC22 but not with the PC20. Comparison of diagnostic accuracy between two needles (PC20 vs. PC22, PC20 vs. AC22 and $\mathrm{PC} 22$ vs. AC22) are shown in Additional file 1: Table S1. Meanwhile, the diagnostic accuracy in each pancreatic location tended to be better in $\mathrm{PC} 20$ and AC22 compared to PC22 (Additional file 2: Table S2). Next, we examined the diagnostic efficacy of the first pass. The histological accuracy in the first pass was 85.6 (95/111) with the PC20, 33.8\% (23/68) with the PC22, and $66.7 \%(8 / 12)$ with the AC22 (Table 3). The accuracy was significantly higher with the PC20 than PC22 ( $p$ $\operatorname{adj}<0.0001)$, while there was no significant difference between the PC20 and AC22 ( $p$-adj=0.32). The proportion of adequate specimens by MOSE among the samples from the first pass was $63.9 \%(71 / 111)$ with the PC20, 41.2\% (28/68) with the PC22, and 50.0\% (6/12) with the AC22. There was no significant difference among the needles $(p=0.20)$ (Table 3, Additional file 3: Table S3). The histological accuracy among adequate specimens and clotted specimens was $81.7 \%$ and $92.5 \%$ with the PC20, 39.3\% and $30.0 \%$ with the $\mathrm{PC} 22$, and 83.3\% and $50.0 \%$ with the AC22. 
Table 3 Histological accuracy and adequacy in macroscopic on-site evaluation of specimens from $1 \mathrm{st}$ pass

\begin{tabular}{clllc}
\hline & PC20 & PC22 & AC22 & p value \\
\hline Histological & $85.6 \% \%^{+++}$ & $33.8 \%$ & $66.7 \%$ & $<0.0001^{\#}$ \\
accuracy & $(95 / 111)$ & $(23 / 68)$ & $(8 / 12)$ & \\
Macroscopic & $63.9 \%$ & $41.2 \%$ & $50.0 \%$ & $0.20^{\#}$ \\
$\begin{array}{c}\text { on-site evalu- } \\
\text { ation }\end{array}$ & $(71 / 111)$ & $(28 / 68)$ & $(6 / 12)$ & \\
\hline
\end{tabular}

PC20 EchoTip ProCore 20G, PC22 EchoTip ProCore 22G, AC22 Acquire 22G

\# Chi-squared test among PC20, PC22, and AC22

tttt Adjusted $p<0.0001$, Fisher's exact test followed by Bonferroni correction; $P C 20$ versus $P C 22$

Table 4 The cases using additional needles

\begin{tabular}{|c|c|c|}
\hline & Number of cases" & Reason of using additional needles \\
\hline PC20 & $5(4.5 \% ; 5 / 111)$ & $\begin{array}{l}\text { The needle got distorted and the } \\
\text { stylet could not be inserted: } 2 \text { cases } \\
\text { Insufficient specimens: } 3 \text { cases }\end{array}$ \\
\hline PC22 & $7(10.3 \% ; 7 / 68)$ & Insufficient specimens: 7 cases \\
\hline AC22 & $2(16.7 \% ; 2 / 12)$ & Insufficient specimens: 2 cases \\
\hline
\end{tabular}

PC20 EchoTip ProCore 20G, PC22 EchoTip ProCore 22G, AC22 Acquire 22G

\# $p=0.20$, Chi-squared test among PC20, PC22, and AC22

\section{Requirement of additional EUS-FNA/B needles}

Among the 111 cases in which the PC20 was used, 5 (4.5\%) required a biopsy needle change. The needle was distorted and the stylet could not be inserted in two of these five cases. In the other three cases, the needles were changed because of insufficient specimens (i.e., fragment specimens by MOSE). The PC22 was changed in 7 cases because of fragment specimens $(10.3 \%, 7 / 68)$. Two cases in which the $\mathrm{AC} 22$ was used required a needle change because of fragment specimens $(16.7 \%, 2 / 12)$. There was no significant difference in the requirement of a needle change among the different needle types $(p=0.20)$ (Table 4).

\section{Adverse events related to EUS-FNA/B procedure}

One patient in the PC20 group developed mild pancreatitis $(0.9 \%, 1 / 111)$. The patient recovered with conservative treatment (fasting, fluid replacement, protease inhibitor, and antibiotic therapy). No adverse events occurred among the patients in the PC22 group $(0.0 \%, 0 / 68)$ or AC22 group $(0.0 \%, 0 / 12)(p=0.54)$.

\section{Discussion}

In this retrospective study, we examined the diagnostic efficacy of three puncture needles (PC20, PC22, and $\mathrm{AC} 22$ ) for the first EUS-FNA/B in patients with pancreatic cancer. The PC20 and PC22 employ a core trap system, which contributes to increased tissue acquisition and preservation of the histological structure [23]. A core trap can reportedly ensure effective cellularity for diagnosis [21, 24]. Acquiring tissue specimens with minimal structural damage increases the histopathological quality of the samples. High-quality specimens are crucial for assessment of the subtypes of malignancy (e.g., adenocarcinoma, adenosquamous carcinoma, anaplastic carcinoma, neuroendocrine carcinoma, and metastatic carcinoma) and performance of immunohistochemistry $[25,26]$. Therefore, a core trap is an advantage in histopathological diagnosis of pancreatic tumors by EUSFNA/B. However, although both the PC20 and PC22 have a core trap, the present study demonstrated that diagnostic accuracy was significantly higher with the PC20 than PC22. Two possible factors led to the difference in diagnostic accuracy between the PC20 and PC22: (1) the difference in the needle gauge and (2) the difference in the design of the core trap. A thicker needle is reportedly more useful for collection of core tissue [27] and preservation of the histological structure of specimens [18] than is a thinner needle. Meanwhile, Armellini et al. [28] reported that the forward-bevel core trap (PC20) enables the acquisition of more tissue than the reverse-bevel core trap (PC22). This is thought to be because the forwardbevel core trap catches the tissue while the needle moves forward [28]. Additionally, the core trap is larger in the PC20 than PC22 (2.9 vs. $2.0 \mathrm{~mm}$, respectively). However, there is little difference in the distance from the tip to the core trap between the PC20 and PC22 (3.8 vs. $3.9 \mathrm{~mm}$, respectively). This suggests that the stroke length measured by EUS imaging does not drive the needle choice between the PC20 and PC22. Our findings suggest that the PC20 is preferable for the histological diagnosis. The total accuracy (combined histology and cytology) with the PC20 (96.4\%) was equal to the histological result; i.e., there was no additional diagnostic impact of cytology. Additionally, even in samples defined as clotted specimens by MOSE, the histological accuracy of clotted specimens was $92.5 \%$ with the PC20. In one study, the histological sensitivity was $92.4 \%$ in whitish tissue and $40.8 \%$ in blood clots [29]. Although a sample obtained by a thicker needle is contaminated by more blood [30], our results suggest that clotted specimens obtained by the PC20 can still be used for histological assessment.

We also assessed the diagnostic accuracy of the AC22 in this study. The AC22 is a relatively new needle with a diagnostic accuracy of 94.0-96.7\% [31-33]. Ishikawa et al. [34] reported that the AC22 was better than a conventional needle in acquiring cells. The AC22 is designed to hold and cut the tissue with multiple sharp structures on the tip (fork tip). This characteristic structure contributes to better sample collection even in a lesion 
with dense fibrotic change, different from most conventional EUS-FNA/B needles [32, 35]. A randomized trial by Bang et al. [36]. demonstrated that AC22 showed better diagnostic accuracy for pancreatic masses compared to 22-gauge conventional needles and PC22 In an animal experiment, there was no significant difference in the core tissue acquisition ability between the PC20 and AC22 [22]. Considering that the AC22 is thinner (22G) than the PC20 (20G), the fork-tip structure of the AC22 could be more advantageous for tissue collection than the core trap of the PC20. However, a recent study proposed that the side bevel, which moves to and fro in the tumor during the sampling procedure, can cut the tumor surface more effectively than the needle tip within the tumor [37]. Thus, considering the tip structure of the AC22, this needle might be more effective than other types particularly for cases in which a long puncture stroke length cannot be secured. Fujita et al. [38] compared the diagnostic accuracy between the AC22 and a conventional needle and found no difference between the two needles for $>20$-mm tumors; however, there was a significant difference for $<19$-mm tumors. There was no significant difference in the histological accuracy between the PC20 and AC22 in the present study $(p=0.02, p$-adj $=0.06)$. A sufficient stroke length was secured for all patients; however, given that the puncture stroke length was significantly shorter with the AC22 than PC20, it is difficult to draw a strict conclusion regarding the efficacy of the PC20 versus AC22 from this study. However, our findings suggest that as long as a sufficient stroke length can be secured, the diagnostic accuracy of both the PC20 and AC22 is sufficiently high. Given our results that the histological accuracy tended to be higher with the PC20 than AC22 as well as the small number of patients in whom the $\mathrm{AC} 22$ was used, we speculate that the PC20 is suitable for cases in which histological assessment including immunohistochemistry can be critical for diagnosis, while the AC22 is suitable for small tumors in which it is difficult to secure a long stroke length.

In EUS-FNA/B, transduodenal puncture is challenging in some cases because of the steep up-angle of the scope $[23,39]$. A thicker needle with less flexibility has a disadvantage in this procedure, and endoscopists need to use additional needles in some cases. In this study, however, the operability of the PC20 was not different from that of the PC22 and AC22. This finding suggests that the PC20 can be used for transduodenal puncture similarly to $22 \mathrm{G}$ needles. In terms of adverse events, one case of mild pancreatitis was observed in the PC20 group (0.9\%), and no adverse events occurred in the PC22 and AC22 groups. No serious complications such as active bleeding or perforation occurred with any needles. A systematic review on the safety of EUS-FNA revealed a complication rate of $0.98 \%$ and mortality rate of $0.02 \%$ [ 40 ]. The needle diameter is not thought to be associated with the adverse event rate [41, 42]. Considering the results of both our study and previous studies, it seems that there is no considerable difference in safety among the PC20, PC22, and AC22.

This study had several limitations. First, because it was a single-center, retrospective study, the EUS-FNA/B skills might have varied over time and/or among endoscopists, and the needle selection by each operator might have been biased. There could be a possibility that the characteristic structure of $\mathrm{AC} 22$, the multiple-sharp-structure tip, impacts the needle selection. Second, the AC22 was approved for EUS-FNA/B for pancreatic solid lesions in October 2016 in Japan, and in our facility, the AC22 has been used in much fewer patients than the PC20 and PC22. It is needed to perform a prospective study with randomizing the needle selection and setting the specific criteria for the tumor size and the number of passes. Additionally, in this study, the stroke length of puncture under EUS images was not controlled between the PC20 and AC22. A study in which the stroke length is identical between the needles is necessary for direct comparison of the efficacy of these needles.

\section{Conclusions}

In conclusion, this study demonstrated that the PC20 has better diagnostic efficacy than the PC22. Although further study is needed to compare the PC20 and AC22, the high histological accuracy of the PC20 could be advantageous for lesions in which histological assessment is critical, while the AC22 could be a good option particularly for small lesions. Evidence-based needle selection could contribute to the improvement of diagnosing pancreatic cancer.

\section{Supplementary Information}

The online version contains supplementary material available at https://doi. org/10.1186/s12876-020-01583-7.

Additional file 1. Table S1. Comparison of diagnostic accuracy between two needles.

Additional file 2. Table S2. Comparison of diagnostic accuracy in each pancreatic location between two needles.

Additional file 3. Table S3. Comparison of macroscopic on-site evaluation between two needles.

\section{Abbreviations}

AC22: Acquire 22G $\mathrm{G}^{\circledR}$; EUS-FNA/B: Endoscopic ultrasound-guided fine-needle aspiration/biopsy; MOSE: Macroscopic on-site evaluation; PC20: EchoTip ProCore 20G ${ }^{\circledR} ;$ PC22: EchoTip ProCore 22G ${ }^{\circledR}$.

\section{Acknowledgements}

We thank Angela Morben, DVM, ELS, from Edanz Group (https://en-autho $r$-services.edanzgroup.com/), for editing a draft of this manuscript. 


\section{Authors' contributions}

SW, JM, and TH designed and conceived this study. SW, JM, and TH analyzed and interpreted the results and prepared the manuscript. SW, MT, KK, SK, TN, $\mathrm{TG}, \mathrm{HO}, \mathrm{KO}, \mathrm{KG}, \mathrm{NI}, \mathrm{ST}, \mathrm{KN}$, and JS collected and analyzed the data. JM and TH oversaw the entire project. TH approved the final draft submitted. All authors read and approved the final manuscript.

\section{Funding}

Not applicable.

\section{Availability of data and materials}

The datasets used and analyzed during the current study are available from the corresponding author on reasonable request.

\section{Ethics approval and content to participate}

The present study was approved by the Institutional Review Board of Kyorin University School of Medicine (IRB No. 1099) on 27 March 2018. This study was performed in accordance with the guidelines of the Declaration of Helsinki. The IRB approved the use of an opt-out method of consent and informed consent was obtained using an opt-out method with the written document on the research.

\section{Consent for publication}

By opt-out of the research, we disclose the information on the bulletin board or website of the facility, guarantee the opportunity for the research subject to refuse to participate in the research, and provide a contact point (contact address) that accepts the intention to reject the research.

\section{Competing interests}

The authors declare that they have no competing interests.

\section{Author details}

${ }^{1}$ Department of Gastroenterology and Hepatology, Kyorin University School of Medicine, 6-20-2 Shinkawa, Mitaka-shi, Tokyo 181-8611, Japan. ${ }^{2}$ Department of Gastroenterology and Hepatology, Tokyo Medical University, 6-7-1 Nishishinjuku, Shinjuku-ku, Tokyo 160-0023, Japan. ${ }^{3}$ Department of Gastroenterology and Hepatology, Tokyo Dental College Ichikawa General Hospital, 5-11-13 Sugano, Ichikawa-shi, Chiba 272-8513, Japan. ${ }^{4}$ Department of Pathology, Kyorin University School of Medicine, 6-20-2 Shinkawa, Mitaka-shi, Tokyo 181-8611, Japan.

Received: 10 August 2020 Accepted: 15 December 2020 Published online: 06 January 2021

\section{References}

1. Siegel R, Ma J, Zou Z, Jemal A. Cancer statistics, 2014. CA Cancer J Clin. 2014;64:9-29.

2. Gillen S, Schuster T, Zum Buschenfelde CM, Friess H, Kleeff J. Preoperative/neoadjuvant therapy in pancreatic cancer: a systematic review and meta-analysis of response and resection percentages. PLoS Med. 2010;7:1000267.

3. Ilic M, Ilic I. Epidemiology of pancreatic cancer. World J Gastroenterol. 2016;22:9694-705.

4. Luo J, Xiao L, Wu C, Zheng Y, Zhao N. The incidence and survival rate of population-based pancreatic cancer patients: Shanghai Cancer Registry 2004-2009. PLOS ONE. 2013;8:e76052.

5. American Cancer Society. Key statistics for pancreatic cancer. https:// www.cancer.org/cancer/pancreatic-cancer/about/key-statistics.html. Accessed 17 April 2020

6. Saad AM, Turk T, Al-Husseini MJ, Abdel-Rahman O. Trends in pancreatic adenocarcinoma incidence and mortality in the United States in the last four decades; a SEER-based study. BMC Cancer. 2018;18:688.

7. Pourshams A, Sepanlou SG, Ikuta KS, Bisignano C, Safiri S, Roshandel G, et al. The global, regional, and national burden of pancreatic cancer and its attributable risk factors in 195 countries and territories, 1990-2017: a systematic analysis for the Global Burden of Disease Study 2017. Lancet Gastroenterol Hepatol. 2019;4:934-47.
8. McGuigan A, Kelly P, Turkington RC, Jones C, Coleman HG, McCain RS Pancreatic cancer: a review of clinical diagnosis, epidemiology, treatment and outcomes. World J Gastroenterol. 2018;24:4846-61.

9. Parkin DM, Boyd L, Walker LC. The fraction of cancer attributable to lifestyle and environmental factors in the UK in 2010. Br J Cancer. 2011; 105(Suppl 2):S2-5.

10. Gowing LR, Ali RL, Allsop S, Marsden J, TurfEE, West R, et al. Global statistics on addictive behaviours: 2014 status report. Addiction. 2015;110:904-19.

11. Facciorusso A, Martina M, Buccino RV, Nacchiero MC, Muscatiello N. Diagnostic accuracy of fine-needle aspiration of solid pancreatic lesions guided by endoscopic ultrasound elastography. Ann Gastroenterol. 2018:31:513-8.

12. Wang M, Huang S, Pei R, Lin J, Yang X. Endoscopic ultrasonography guided transgastric trans-portal system fine needle aspiration for diagnosing pancreatic head and uncinate process malignancy. Ann Transl Med. 2019;7:719.

13. Kim J, Ryu JK, Park JM, Paik WH, Song BJ, Kim YT, et al. Clinical factors associated with accuracy of EUS-FNA for pancreatic or peripancreatic solid mass without on-site cytopathologists. J Gastroenterol Hepatol. 2014;29:887-92.

14. Madhoun MF, Wani SB, Rastgi A, Early D, Gaddam S, Tierney WM, et al. The diagnostic accuracy of 22-gauge and 25-gauge needles in endoscopic ultrasound-guided fine needle aspiration of solid pancreatic lesions: a meta-analysis. Endoscopy. 2013;45:86-92.

15. Haba S, Yamao K, Bhatia V, Mizuno N, Hara K, Hijioka S, et al. Diagnostic ability and factors affecting accuracy of endoscopic ultrasound-guided fine needle aspiration for pancreatic solid lesions: Japanese large single center experience. J Gastroenterol. 2013;48:973-81.

16. Siddiqui UD, Rossi F, Rosenthal LS, Padda MS, Murali-Dharan V, Aslanian HR. EUS-guided FNA of solid pancreatic masses: a prospective, randomized trial comparing 22-gauge and 25-gauge needles. Gastrointest Endosc. 2009;70:1093-7.

17. YusufTE, Ho S, Pavey DA, Michael H, Gress FG. Retrospective analysis of the utility of endoscopic ultrasound-guided fine-needle aspiration (EUSFNA) in pancreatic masses, using a 22-gauge or 25-gauge needle system: a multicenter experience. Endoscopy. 2009;41:445-8.

18. Song TJ, Kim JH, Lee SS, Eum JB, Moon SH, Park DH, et al. The prospective randomized, controlled trial of endoscopic ultrasound-guided fine-needle aspiration using $22 \mathrm{G}$ and $19 \mathrm{G}$ aspiration needles for solid pancreatic or peripancreatic masses. Am J Gastroenterol. 2010;105:1739-45.

19. Li H, Li W, Zhou QY, Fan B. Fine needle biopsy is superior to fine needle aspiration in endoscopic ultrasound guided sampling of pancreatic masses. Medicine. 2018;97(13):e0207.

20. Matsubayashi H, Matsui T, Yabuuchi Y, Imai K, Tanaka M, Kakushima N, et al. Endoscopic ultrasonography guided-fine needle aspiration for the diagnosis of solid pancreaticobiliary lesions: clinical aspects to improve the diagnosis. World J Gastroenterol. 2016;22:628-40.

21. Sterlacci W, Sioulas AD, Veits L, Gonullu P, Schachschal G, Groth S, et al. 22-Gauge core vs 22-gauge aspiration needle for endoscopic ultrasound-guided sampling of abdominal masses. World J Gastroenterol. 2016;22:8820-30

22. Mukai S, Itoi T, Katanuma A, Irisawa A. An animal experimental study to assess the core tissue acquisition ability of endoscopic ultrasoundguided histology needles. Endosc Ultrasound. 2018;7:263-9.

23. Conti CB, Cereatti F, Grassia R. Endoscopic ultrasound-guided sampling of solid pancreatic masses: the fine needle aspiration or fine needle biopsy dilemma. Is the best needle yet to come? World J Gastrointest Endosc. 2019;11:454-71.

24. Alatawi A, Beuvon F, Grabar S, Leblanc S, Chaussade S, Terris B, et al. Comparison of $22 \mathrm{G}$ reverse-beveled versus standard needle for endoscopic ultrasound-guided sampling of solid pancreatic lesions. United Eur Gastroenterol J. 2015;3:343-52.

25. Varadarajulu S, Fraig M, Schmulewitz N, Roberts S, Wildi S, Hawes RH, et al Comparison of EUS-guided 19-gauge Trucut needle biopsy with EUSguided fine-needle aspiration. Endoscopy. 2004;36:397-401.

26. Levy MJ, Jondal ML, Clain J, Wiersema MJ. Preliminary experience with an EUS-guided trucut biopsy needle compared with EUS-guided FNA. Gastrointest Endosc. 2003;57:101-6.

27. Iwashita T, Nakai Y, Samarasena JB, Park DH, Zhang Z, Gu M, et al. High single-pass diagnostic yield of a new 25-gauge core biopsy needle for 
EUS-guided FNA biopsy in solid pancreatic lesions. Gastrointest Endosc. 2013;77:909-15.

28. Armellini E, Manfrin E, Trisolini E, Andorno S, Ballare M, Bernardoni L, et al. Histologic retrieval rate of a newly designed side-bevelled $20 \mathrm{G}$ needle for EUS-guided tissue acquisition of solid pancreatic lesions. United Eur Gastroenterol J. 2019;7:96-104.

29. Iwashita T, Yasuda I, Mukai T, Doi S, Nakashima M, Uemura S, et al. Macroscopic on-site quality evaluation of biopsy specimens to improve the diagnostic accuracy during EUS-guided FNA using a 19-gauge needle for solid lesions: a single-center prospective pilot study (MOSE study). Gastrointest Endosc. 2015;81:177-85.

30. Yang MJ, Yim H, Hwang JC, Lee D, Kim YB, Lim SG, et al. Endoscopic ultrasound-guided sampling of solid pancreatic masses: 22-gauge aspiration versus 25-gauge biopsy needles. BMC Gastroenterol. 2015;15:122.

31. Bang JY, Hebert-Magee S, Navaneethan U, Hasan MK, Hawes R, Varadarajulu S. Randomized trial comparing the Franseen and Fork-tip needles for EUS-guided fine-needle biopsy sampling of solid pancreatic mass lesions. Gastrointest Endosc. 2018;87:1432-8.

32. Mita N, Iwashita T, Uemura S, I wasa Y, Toda K, Mukai T, et al. Endoscopic ultrasound-guided fine needle biopsy using 22-gauge Franseen needle for the histological diagnosis of solid lesions: a multicenter prospective pilot study. Dig Dis Sci. 2020;65:1155-63.

33. Bang JY, Hebert-Magee S, Hasan MK, Navaneethan U, Hawes R, Varadarajulu S. Endoscopic ultrasonography-guided biopsy using a Franseen needle design: initial assessment. Dig Endosc. 2017;29:338-46.

34. Ishikawa T, Kawashima H, Ohno E, Tanaka H, Sakai D, lida T, et al. Clinical impact of EUS-guided fine needle biopsy using a novel Franseen needle for histological assessment of pancreatic diseases. Can J Gastroenterol Hepatol. 2019;2019:8581743.

35. Iwashita T, Yasuda I, Shimizu M. Endoscopic ultrasound-guided tissue acquisition: can fork and crown cut the tissue? Dig Endosc. 2019;31:146-7.

36. Bang JY, Krall K, Hjala N, Singh C, Tejani M, Arnoletti JP, et al. Comparing needle and methods of endoscopic ultrasound-guided fine-needle biopsy to optimize specimen quality and diagnostic accuracy for patients with pancreatic masses in a randomized trial. Clin Gastroenterol Hepatol. 2020;8:S1542-3565.

37. Cheng B, Zhang Y, Chen Q, Sun B, Deng Z, Shan H, et al. Analysis of fineneedle biopsy versus fine-needle aspiration in diagnosis of pancreatic and abdominal masses: a prospective, multicenter, randomized controlled trial. Clin Gastroenterol Hepatol. 2018;16:1314-21.

38. Fujita A, Ryozawa S, Mizuide M, Araki R, Nagata K, Tnisaka Y, et al. Does endoscopic ultrasound-guided fine needle biopsy using a Franseen needle really offer high diagnostic accuracy? A propensity-matched analysis. Endosc Int Open. 2019;7:E1327-32.

39. Iwashita T, Nakai Y, Mukai T, Togawa O, Matsubara S, Hatano Y, et al. A 19-gauge histology needle versus a 19-gauge standard needle in endoscopic ultrasound-guided fine-needle aspiration for solid lesions: a multicenter randomized comparison study (GREATER Study). Dig Dis Sci. 2018;63:1043-51.

40. Wang KX, Ben QW, Jin ZD, Du YQ, Zou DW, Liao Z, et al. Assessment of morbidity and mortality associated with EUS-guided FNA: a systematic review. Gastrointest Endosc. 2011;73:283-90.

41. Varadarajulu S, Ginnetti L, Peetermans J, Rousseau M, Hasan M, Hawes R. Meta-analysis comparing ratesof complications betweenthe standard19Gand22G/25G needlesfor EUS-guidedFNAof pancreatic lesions. Gastrointest Endosc. 2013;77:AB405.

42. Polkowski M, Jenssen C, Kaye P, Carrara S, Deprez P, Gines A, et al. Technical aspects of endoscopic ultrasound (EUS)-guided sampling in gastroenterology: European Society of Gastrointestinal Endoscopy (ESGE) Technical Guideline- March 2017. Endoscopy. 2017;49:989-1006.

\section{Publisher's Note}

Springer Nature remains neutral with regard to jurisdictional claims in published maps and institutional affiliations.
Ready to submit your research? Choose BMC and benefit from:

- fast, convenient online submission

- thorough peer review by experienced researchers in your field

- rapid publication on acceptance

- support for research data, including large and complex data types

- gold Open Access which fosters wider collaboration and increased citations

- maximum visibility for your research: over $100 \mathrm{M}$ website views per year

At BMC, research is always in progress.

Learn more biomedcentral.com/submissions 